Groups Geom. Dyn. 4 (2010), 407-417

DOI $10.4171 / \mathrm{GGD} / 88$
Groups, Geometry, and Dynamics

(C) European Mathematical Society

\title{
A recursive presentation for Mihailova's subgroup
}

\author{
Oleg Bogopolski and Enric Ventura
}

\begin{abstract}
An explicit recursive presentation for Mihailova's subgroup $M(H)$ of $F_{n} \times F_{n}$ corresponding to a finite, concise and Peiffer aspherical presentation $H=\left\langle x_{1}, \ldots, x_{n}\right|$ $\left.R_{1}, \ldots, R_{m}\right\rangle$ is given. This partially answers a question of R. I. Grigorchuk. As a corollary, we construct a finitely generated recursively presented orbit undecidable subgroup of $\operatorname{Aut}\left(F_{3}\right)$.
\end{abstract}

Mathematics Subject Classification (2010). 20F05, 20 F10.

Keywords. Mihailova's subgroup, recursive presentation, asphericity, orbit undecidability.

\section{Introduction}

For all the paper, let $n \geqslant 2$, let $F_{n}$ be the free group with basis $\left\{x_{1}, \ldots, x_{n}\right\}$, and let $H=\left\langle x_{1}, \ldots, x_{n} \mid R_{1}, \ldots, R_{m}\right\rangle$ be a finite presentation of a quotient $H$ of $F_{n}$ (although most of what follows will depend on the specific presentation, we shall make the usual abuse of notation which consists on denoting by $H$ both the group and its given presentation).

K. A. Mihailova, in her influential paper [12], associated to the presentation $H$ the Mihailova subgroup of $F_{n} \times F_{n}$, namely

$$
M(H)=\left\{\left(w_{1}, w_{2}\right) \in F_{n} \times F_{n} \mid w_{1}={ }_{H} w_{2}\right\} \leqslant F_{n} \times F_{n},
$$

i.e., the subgroup of pairs of words in $F_{n}$ determining the same element in $H$. It is clear that $\left(x_{i}, x_{i}\right)$ and $\left(1, R_{j}\right)$ belong to $M(H)$ for all $i=1, \ldots, n$ and $j=1, \ldots, m$, and it is not difficult to see that, in fact, these pairs generate $M(H)$. The important observation made in [12] says that the membership problem for $M(H)$ in $F_{n} \times F_{n}$ is solvable (i.e., there exists an algorithm to decide whether a given $\left(w_{1}, w_{2}\right) \in F_{n} \times F_{n}$ belongs to $M(H)$ or not) if and only if the word problem for $H$ is solvable.

By a result of P. S. Novikov [14] and W. W. Boone [3] (see also [4]), there exist finitely presented groups with unsolvable word problem. Thus, there also exist finitely generated subgroups of $F_{n} \times F_{n}$ with unsolvable membership problem.

Clearly, $M(H)$ has solvable word problem for every $H$ (because $F_{n} \times F_{n}$ also does). In particular, $M(H)$ is recursively presented. More interestingly, F. J. Grunewald proved, in [9, Theorem B], that if $H$ is infinite then $M(H)$ cannot be finitely 
presented. In [1], G. Baumslag and J. E. Roseblade completely described the structure of finitely presented subgroups of $F_{n} \times F_{n}$, a result that was later reproved by H. Short [15] and M. R. Bridson and D. T. Wise [5], and that implies Grunewald's result.

In this context, a natural problem is to look for recursive presentations for Mihailova's group $M(H)$, in terms of the original presentation $H$. This was recently posted as Problem 4.14 in [8] by R. I. Grigorchuk: "What kind of presentations can be obtained for Mihailova's subgroups of $F_{n} \times F_{n}$ determined by finite automata?"

The main result in the present paper (Theorem 1.1 below) gives a partial answer to this problem: under certain technical conditions on the initial $H$ we give an explicit recursive presentation for $M(H)$ with finitely many generators and a one-parametric family of relations.

Theorem 1.1. Let $F_{n}$ be the free group on $x_{1}, \ldots, x_{n}$, and let $H=\left\langle x_{1}, \ldots, x_{n}\right|$ $\left.R_{1}, \ldots, R_{m}\right\rangle$ be a finite, concise and Peiffer aspherical presentation. Then Mihailova's group $M(H) \leqslant F_{n} \times F_{n}$ admits the following presentation

$$
\left\langle d_{1}, \ldots, d_{n}, t_{1}, \ldots, t_{m} \mid\left[t_{j}, d^{-1} t_{i}^{-1} r_{i} d\right],\left[t_{i}, \operatorname{root}\left(r_{i}\right)\right] \quad\left(1 \leqslant i, j \leqslant m, d \in D_{n}\right)\right\rangle,
$$

where $D_{n}$ is the free group with basis $d_{1}, \ldots, d_{n}$, where $r_{i}$ denotes the word in $D_{n}$ obtained from $R_{i}$ by replacing each $x_{k}$ to $d_{k}$, and where $\operatorname{root}\left(r_{i}\right)$ denotes the unique element $s_{i} \in D_{n}$ such that $r_{i}$ is a positive power of $s_{i}$ but $s_{i}$ itself is not a proper power.

In this presentation the elements $d_{i}$ and $t_{j}$ correspond, respectively, to the elements $\left(x_{i}, x_{i}\right)$ and $\left(1, R_{j}\right)$ of $M(H)$.

As a corollary we deduce the existence of a finitely generated, orbit undecidable subgroup of $\operatorname{Aut}\left(F_{3}\right)$ (see [2] for details), which has the recursive presentation given in Theorem 1.1. Another application of Mihailova's construction can be found in [10], Proposition 5.4.

The structure of the paper is as follows. In Section 2 we recall some definitions and discuss some properties of concise and Peiffer aspherical presentations that will be used later. In Section 3 we prove Theorem 1.1. And in Section 4 we recall the relationship between Mihailova's subgroup and orbit undecidability, recently discovered in [2], and deduce the announced corollary (Theorem 4.2).

\section{Asphericity}

As stated, let $\left\langle x_{1}, \ldots, x_{n} \mid R_{1}, \ldots, R_{m}\right\rangle$ be a finite presentation. Formally, $R_{1}, \ldots$, $R_{m}$ is a list of words in the alphabet $\left\{x_{1}, \ldots, x_{n}\right\}^{ \pm 1}$ which may contain trivial and non-reduced words, possible repetitions, and even possible members conjugated to each other or to the inverse of each other. 
A presentation $\left\langle x_{1}, \ldots, x_{n} \mid R_{1}, \ldots, R_{m}\right\rangle$ is called concise if every relation $R_{i}$ is non-trivial and reduced, and every two relations $R_{i}, R_{j}, i \neq j$, are not conjugate to each other, or to the inverse of each other. Given an arbitrary finite presentation, $\left\langle x_{1}, \ldots, x_{n} \mid R_{1}, \ldots, R_{m}\right\rangle$, one can always reduce the relations and eliminate some of them, to obtain another presentation of the same group, which is concise. We call this a concise refinement of $\left\langle x_{1}, \ldots, x_{n} \mid R_{1}, \ldots, R_{m}\right\rangle$.

Now we recall the definition of Peiffer transformations. Consider some elements $U_{1}, \ldots, U_{l} \in F_{n}$, some relators $R_{i_{1}}, \ldots, R_{i_{l}} \in\left\{R_{1}, \ldots, R_{n}\right\}$, and some numbers $\varepsilon_{1}, \ldots, \varepsilon_{l} \in\{-1,1\}$ such that the equation

$$
\left(U_{1} R_{i_{1}}^{\varepsilon_{1}} U_{1}^{-1}\right) \ldots\left(U_{l} R_{i_{l}}^{\varepsilon_{l}} U_{l}^{-1}\right)=1
$$

holds in $F_{n}$. In this situation, the sequence of elements $\left(U_{1} R_{i_{1}}^{\varepsilon_{1}} U_{1}^{-1}, \ldots, U_{l} R_{i_{l}}^{\varepsilon_{l}} U_{l}^{-1}\right)$ of $F_{n}$ is called an identity among relations of length $l$. For $l=0$ we have the empty identity among relations, ( ).

In such a sequence, let us replace two consecutive terms, say $U_{p} R_{i_{p}}^{\varepsilon_{p}} U_{p}^{-1}$ and $U_{p+1} R_{i_{p+1}}^{\varepsilon_{p+1}} U_{p+1}^{-1}$ for some $1 \leqslant p \leqslant l-1$, by the new ones $U_{p+1} R_{i_{p+1}}^{\varepsilon_{p+1}} U_{p+1}^{-1}$ and $\left(U_{p+1} R_{i_{p+1}}^{-\varepsilon_{p+1}} U_{p+1}^{-1} U_{p}\right) R_{i_{p}}^{\varepsilon_{p}}\left(U_{p}^{-1} U_{p+1} R_{i_{p+1}}^{\varepsilon_{p+1}} U_{p+1}^{-1}\right)$. Since the product of the two old terms do coincide with that of the two new ones, the new sequence is again an identity among relations. This transformation is called a Peiffer transformation of the first kind or, shortly, an exchange.

Suppose now that in the sequence $\left(U_{1} R_{i_{1}}^{\varepsilon_{1}} U_{1}^{-1}, \ldots, U_{l} R_{i_{l}}^{\varepsilon_{l}} U_{l}^{-1}\right)$ there are two consecutive terms, say $U_{p} R_{i_{p}}^{\varepsilon_{p}} U_{p}^{-1}$ and $U_{p+1} R_{i_{p+1}}^{\varepsilon_{p+1}} U_{p+1}^{-1}$ for some $1 \leqslant p \leqslant l-1$, whose product equals 1 . Then we can obtain a new identity among relations by just deleting these two terms. This transformation and the inverse one are called Peiffer transformations of the second kind or shortly, deletion and insertion, respectively.

Definition 2.1. We say that a presentation is Peiffer aspherical if every identity among relations can be carried to the empty one by a sequence of Peiffer transformations.

In particular, a presentation admitting identities among relations of odd length is automatically not Peiffer aspherical.

A large class of Peiffer aspherical presentations can be obtained by using Theorems 3.1 and 4.2, and Lemma 5.1 from [6]. They state, respectively, that Peiffer asphericity is preserved under certain HNN extensions, under free products, and under Tietze transformations.

In the next section we shall argue using Peiffer asphericity. However, for completeness, we mention that in the literature there are (at least) three concepts of asphericity for presentations, which do not agree in general: Peiffer asphericity (called combinatorial asphericity in [6], see Proposition 1.5 there); diagrammatical asphericity defined in [6] like Peiffer asphericity but without allowing insertions (and also considered in Chapter III.10 of [11]); and topological asphericity. 
Let $H=\left\langle x_{1}, \ldots, x_{n} \mid R_{1}, \ldots, R_{m}\right\rangle$ be a presentation and $\mathcal{K}(H)$ be the twodimensional $\mathrm{CW}$-complex with a single 0 -cell, $n$ 1-cells corresponding to the generators $x_{1}, \ldots, x_{n}$, and $m$ 2-cells each one being attached to the 1-skeleton along the path determined by the spelling of the corresponding relation. The presentation $H$ is said to be topologically aspherical if $\pi_{2}(\mathcal{K}(H))=0$. As is indicated in Proposition 1.1 of [6], this is equivalent to the triviality of the second homology group of the universal cover of $\mathcal{K}(H)$.

The relations between these three concepts are as follows (for more details, see the introduction and Proposition 1.3 of [6]):

(i) topological asphericity implies Peiffer asphericity,

(ii) diagrammatical asphericity implies Peiffer asphericity,

(iii) for presentations where every relation is reduced, topological asphericity is equivalent to Peiffer asphericity plus conciseness and "no relator being a proper power".

\section{Proof of Theorem 1.1}

Back to Mihailova's construction for $H=\left\langle x_{1}, \ldots, x_{n} \mid R_{1}, \ldots, R_{m}\right\rangle$, we recall that $M(H) \leqslant F_{n} \times F_{n}$ is generated by $\left(x_{i}, x_{i}\right)$ and $\left(1, R_{j}\right), i=1, \ldots, n, j=1, \ldots, m$. So, letting $F_{n+m}$ be the free group with basis $\left\{d_{1}, \ldots, d_{n}, t_{1}, \ldots, t_{m}\right\}$, we have an epimorphism $\pi: F_{n+m} \rightarrow M(H)$ defined by $d_{i} \mapsto\left(x_{i}, x_{i}\right)$ and $t_{j} \mapsto\left(1, R_{j}\right)$, $i=1, \ldots, n, j=1, \ldots, m$. Now, for proving Theorem 1.1 we have to show that ker $\pi$ is precisely the normal closure of the relations shown in the pretended presentation for $M(H)$. Note that the images of elements $d_{1}, \ldots, d_{n}$ generate the diagonal subgroup of $F_{n} \times F_{n}$, denoted $\operatorname{Diag}\left(F_{n} \times F_{n}\right)$, which is isomorphic to $F_{n}$; hence, $\pi$ restricts to an isomorphism from $D_{n}=\left\langle d_{1}, \ldots, d_{n}\right\rangle \leqslant F_{n+m}$ onto $\operatorname{Diag}\left(F_{n} \times F_{n}\right) \leqslant M(H) \leqslant F_{n} \times F_{n}$.

We will keep the following notational convention in the proof: capital letters will always mean words in $x_{1}, \ldots, x_{n}$, like $R_{j}$; with this in mind, if $u$ is a word on $d_{1}, \ldots, d_{n}$, then its capitalization $U$ will denote the word obtained from $u$ by replacing each occurrence of $d_{i}$ to $x_{i}$. Thus, $U$ is just the projection of $\pi(u)$ to the first (or the second) coordinate.

Proof of Theorem 1.1. Recall that in the statement, $r_{j}$ is the word in $D_{n}$ obtained from $R_{j}$ by replacing each $x_{i}$ to $d_{i}, j=1, \ldots, m$.

Let $\mathcal{N}$ be the normal closure (in the free group $F_{n+m}$ ) of the recursive family of commutators

$$
\left\{\left[t_{j}, d^{-1} t_{i}^{-1} r_{i} d\right],\left[t_{i}, \operatorname{root}\left(r_{i}\right)\right] \mid i, j=1, \ldots, m, d \in D_{n}\right\} .
$$


Our goal is to show that $\mathcal{N}=\operatorname{ker} \pi$. The inclusion $\mathcal{N} \leqslant \operatorname{ker} \pi$ is straightforward from the following computations:

$$
\begin{aligned}
\pi\left(\left[t_{j}, d^{-1} t_{i}^{-1} r_{i} d\right]\right) & =\left[\left(1, R_{j}\right),(u, u)^{-1}\left(R_{i}, 1\right)(u, u)\right] \\
& =\left[\left(1, R_{j}\right),\left(u^{-1} R_{i} u, 1\right)\right]=(1,1), \\
\pi\left(\left[t_{i}, \operatorname{root}\left(r_{i}\right)\right]\right) & =\left[\left(1, R_{i}\right),\left(\operatorname{root}\left(R_{i}\right), \operatorname{root}\left(R_{i}\right)\right)\right]=(1,1) .
\end{aligned}
$$

In order to prove ker $\pi \leqslant \mathcal{N}$, we shall use the following strategy: to each word $w \in$ ker $\pi$ we will associate an identity among relations for the presentation $\left\langle x_{1}, \ldots, x_{n}\right|$ $\left.R_{1}, \ldots, R_{m}\right\rangle$ of $H$, in such a way that if $w \neq 1$ then the associated identity is nonempty; then we will show that, after applying an arbitrary Peiffer transformation, the resulting identity among relations is again the one associated to some other word $w^{\prime} \in \operatorname{ker} \pi$ satisfying, additionally, that $w^{-1} w^{\prime} \in \mathcal{N}$.

Having seen this, let $w \in \operatorname{ker} \pi$ and consider the associated identity among relations. Since, by hypothesis, the presentation $\left\langle x_{1}, \ldots, x_{n} \mid R_{1}, \ldots, R_{m}\right\rangle$ is Peiffer aspherical, there exists a sequence of Peiffer transformations reducing such identity to the empty one. Now, repeatedly using the result mentioned in the previous paragraph, we obtain a list of words (ending with the trivial one because the last identity is empty), $w, w^{\prime}, w^{\prime \prime}, \ldots, 1$, and such that the difference between every two consecutive ones belongs to $\mathcal{N}$. This shows that $w \in \mathcal{N}$ concluding the proof.

So we are reduced to construct such an association. Let $w \in \operatorname{ker} \pi \leqslant F_{n+m}$ and write it in the form $w=u_{1} t_{i_{1}}^{\varepsilon_{1}} u_{2} \ldots u_{l} t_{i_{l}}^{\varepsilon_{l}} u_{l+1}$, where $l \geqslant 0$ and $u_{1}, \ldots, u_{l+1}$ are words in $d_{1}, \ldots, d_{n}$. Then, projecting $\pi(w)$ to each coordinate, we have

$$
U_{1} U_{2} \ldots U_{l+1}=1 \quad \text { and } \quad U_{1} R_{i_{1}}^{\varepsilon_{1}} U_{2} \ldots U_{l} R_{i_{l}}^{\varepsilon_{l}} U_{l+1}=1 .
$$

Denote the accumulative products by $\mathbb{U}_{i}=U_{1} U_{2} \ldots U_{i}, i=1, \ldots, l+1$ (note that $\left.\mathbb{U}_{l+1}=1\right)$. By (1), we have

$$
\mathbb{U}_{1} R_{i_{1}}^{\varepsilon_{1}} \mathbb{U}_{1}^{-1} \mathbb{U}_{2} R_{i_{2}}^{\varepsilon_{2}} \mathbb{U}_{2}^{-1} \ldots \mathbb{U}_{l} R_{i_{l}}^{\varepsilon_{l}} \mathbb{U}_{l}^{-1}=1
$$

in the free group $F_{n}$. In other words,

$$
\left(\mathbb{U}_{1} R_{i_{1}}^{\varepsilon_{1}} \mathbb{U}_{1}^{-1}, \mathbb{U}_{2} R_{i_{2}}^{\varepsilon_{2}} \mathbb{U}_{2}^{-1}, \ldots, \mathbb{U}_{l} R_{i_{l}}^{\varepsilon_{l}} \mathbb{U}_{l}^{-1}\right)
$$

is an identity among relations for the presentation $\left\langle x_{1}, \ldots, x_{n} \mid R_{1}, \ldots, R_{m}\right\rangle$ of $H$. This is the identity associated to $w \in \operatorname{ker} \pi$. Note that if this identity is empty, that is $l=0$, then $w=u_{1} \in\left\langle d_{1}, \ldots, d_{n}\right\rangle \cap \operatorname{ker} \pi$ and so $w=1$.

Let us analyze the situation when we apply an arbitrary Peiffer transformation to this identity.

Case 1: Consider the exchange which, for some $1 \leqslant p \leqslant l-1$, replaces the consecutive terms

$$
\mathbb{U}_{p} R_{i_{p}}^{\varepsilon_{p}} \mathbb{U}_{p}^{-1} \quad \text { and } \quad \mathbb{U}_{p+1} R_{i_{p+1}}^{\varepsilon_{p+1}} \mathbb{U}_{p+1}^{-1}
$$


in (2), by the terms

$$
\mathbb{U}_{p+1} R_{i_{p+1}}^{\varepsilon_{p+1}} \mathbb{U}_{p+1}^{-1} \text { and }\left(\mathbb{U}_{p+1} R_{i_{p+1}}^{-\varepsilon_{p+1}} \mathbb{U}_{p+1}^{-1} \mathbb{U}_{p}\right) R_{i_{p}}^{\varepsilon_{p}}\left(\mathbb{U}_{p}^{-1} \mathbb{U}_{p+1} R_{i_{p+1}}^{\varepsilon_{p+1}} \mathbb{U}_{p+1}^{-1}\right)
$$

respectively. We claim that the identity among relations obtained in this way is precisely the one corresponding to the word

$$
w^{\prime}=v_{1} t_{i_{1}}^{\varepsilon_{1}} \ldots v_{p-1} t_{i_{p-1}}^{\varepsilon_{p-1}} v_{p} t_{i_{p+1}}^{\varepsilon_{p+1}} v_{p+1} t_{i_{p}}^{\varepsilon_{p}} v_{p+2} t_{i_{p+2}}^{\varepsilon_{p+2}} \ldots v_{l} t_{i_{l}}^{\varepsilon_{l}} v_{l+1}
$$

where

$$
\begin{array}{ccrlrl}
v_{1} & =u_{1}, & v_{p} & =u_{p} u_{p+1}, & v_{p+3} & =u_{p+3}, \\
\vdots & v_{p+1} & =r_{i_{p+1}}^{-\varepsilon_{p+1}} u_{p+1}^{-1}, & & \vdots \\
v_{p-1} & =u_{p-1}, & v_{p+2} & =u_{p+1} r_{i_{p+1}}^{\varepsilon_{p+1}} u_{p+2}, & v_{l+1} & =u_{l+1} .
\end{array}
$$

And we also claim that $w^{-1} w^{\prime} \in \mathcal{N}$. This second assertion is easy to verify since we can obtain back $w$ from $w^{\prime}$ by permuting the two consecutive subwords $u_{p+1} t_{i_{p+1}}^{\varepsilon_{p+1}} r_{i_{p+1}}^{-\varepsilon_{p+1}} u_{p+1}^{-1}$ and $t_{i_{p}}^{\varepsilon_{p}}$. But the commutator of these two words is an element of $\mathcal{N}$ : for $\varepsilon_{p+1}=-1$ this is immediate; and for $\varepsilon_{p+1}=1$ it follows from the facts that, modulo $\mathcal{N}, t_{i_{p}}$ (and so $t_{i_{p}}^{\varepsilon_{p}}$ ) commutes with $u_{p+1}\left(t_{i_{p+1}}^{-1} r_{i_{p+1}}\right)^{ \pm 1} u_{p+1}^{-1}$, but also $t_{i_{p+1}}$ commutes with $t_{i_{p+1}}^{-1} r_{i_{p+1}}$ (and so, $t_{i_{p+1}}^{-1}$ with $r_{i_{p+1}}$ ). Therefore, $w^{\prime}$ equals $w$ modulo $\mathcal{N}$.

To see the first part of the claim, let us capitalize the $v_{i}$ 's:

$$
\begin{array}{rlrlrl}
V_{1} & =U_{1}, & V_{p} & =U_{p} U_{p+1}, & V_{p+3}=U_{p+3}, \\
& \vdots & V_{p+1} & =R_{i_{p+1}}^{-\varepsilon_{p+1}} U_{p+1}^{-1}, & & \vdots \\
V_{p-1} & =U_{p-1}, & V_{p+2} & =U_{p+1} R_{i_{p+1}}^{\varepsilon_{p+1}} U_{p+2}, & V_{l+1} & =U_{l+1} .
\end{array}
$$

And let us compute the $\mathbb{V}_{i}=V_{1} V_{2} \ldots V_{i}$ 's:

$$
\begin{aligned}
\mathbb{V}_{1} & =\mathbb{U}_{1}, & \mathbb{V}_{p} & =\mathbb{U}_{p+1}, & \mathbb{V}_{p+3} & =\mathbb{U}_{p+3}, \\
& \vdots & \mathbb{V}_{p+1} & =\mathbb{U}_{p+1} R_{i_{p+1}}^{-\varepsilon_{p+1}} \mathbb{U}_{p+1}^{-1} \mathbb{U}_{p}, & & \vdots \\
\mathbb{V}_{p-1} & =\mathbb{U}_{p-1}, & \mathbb{V}_{p+2} & =\mathbb{U}_{p+2}, & \mathbb{V}_{l+1} & =\mathbb{U}_{l+1} .
\end{aligned}
$$


Finally, the identity among relations associated to $w^{\prime}$ is

$$
\begin{aligned}
\left(\mathbb{V}_{1} R_{i_{1}}^{\varepsilon_{1}} \mathbb{V}_{1}^{-1}\right. & =\mathbb{U}_{1} R_{i_{1}}^{\varepsilon_{1}} \mathbb{U}_{1}^{-1}, \\
& \vdots \\
\mathbb{V}_{p-1} R_{i_{p-1}}^{\varepsilon_{p-1}} \mathbb{V}_{p-1}^{-1}= & \mathbb{U}_{p-1} R_{i_{p-1}}^{\varepsilon_{p-1}} \mathbb{U}_{p-1}^{-1}, \\
\mathbb{V}_{p} R_{i_{p+1}}^{\varepsilon_{p+1}} \mathbb{V}_{p}^{-1}= & \mathbb{U}_{p+1} R_{i_{p+1}}^{\varepsilon_{p+1}} \mathbb{U}_{p+1}^{-1}, \\
\mathbb{V}_{p+1} R_{i_{p}}^{\varepsilon_{p}} \mathbb{V}_{p+1}^{-1}= & \mathbb{U}_{p+1} R_{i_{p+1}}^{-\varepsilon_{p+1}} \mathbb{U}_{p+1}^{-1} \mathbb{U}_{p} R_{i_{p}}^{\varepsilon_{p}} \mathbb{U}_{p}^{-1} \mathbb{U}_{p+1} R_{i_{p+1}}^{\varepsilon_{p+1}} \mathbb{U}_{p+1}^{-1}, \\
\mathbb{V}_{p+2} R_{i_{p+2}}^{\varepsilon_{p+2}} \mathbb{V}_{p+2}^{-1}= & \mathbb{U}_{p+2} R_{i_{p+2}}^{\varepsilon_{p+2}} \mathbb{U}_{p+2}^{-1}, \\
& \vdots \\
\mathbb{V}_{l} R_{i_{l}}^{\varepsilon_{l}} \mathbb{V}_{l}^{-1} & \left.=\mathbb{U}_{l} R_{i_{l}}^{\varepsilon_{l}} \mathbb{U}_{l}^{-1}\right),
\end{aligned}
$$

which does coincide with the identity among relations obtained from (2) after applying the Peiffer transformation (3).

Case 2: Consider the deletion which, for some $1 \leqslant p \leqslant l-1$, deletes the consecutive terms

$$
\mathbb{U}_{p} R_{i_{p}}^{\varepsilon_{p}} \mathbb{U}_{p}^{-1} \quad \text { and } \quad \mathbb{U}_{p+1} R_{i_{p+1}}^{\varepsilon_{p+1}} \mathbb{U}_{p+1}^{-1},
$$

in (2), assuming that its product equals 1 . We claim that the identity among relations obtained in this way is precisely the one corresponding to the word

$$
w^{\prime}=v_{1} t_{i_{1}}^{\varepsilon_{1}} \ldots v_{p-1} t_{i_{p-1}}^{\varepsilon_{p-1}} v_{p} t_{i_{p+2}}^{\varepsilon_{p+2}} v_{p+1} \ldots v_{l-2} t_{i_{l}}^{\varepsilon_{l}} v_{l-1},
$$

where

$$
\begin{array}{ccc}
v_{1}=u_{1}, & v_{p+1}=u_{p+3}, \\
\vdots & v_{p}=u_{p} u_{p+1} u_{p+2}, & \vdots \\
v_{p-1}=u_{p-1}, & v_{l-1}=u_{l+1} .
\end{array}
$$

And we also claim that $w^{-1} w^{\prime} \in \mathcal{N}$. This second assertion follows from the hypothesis that $\left(\mathbb{U}_{p} R_{i_{p}}^{\varepsilon_{p}} \mathbb{U}_{p}^{-1}\right) \cdot\left(\mathbb{U}_{p+1} R_{i_{p+1}}^{\varepsilon_{p+1}} \mathbb{U}_{p+1}^{-1}\right)=1$. In fact, conciseness implies that $i_{p}=i_{p+1}, \varepsilon_{p}=-\varepsilon_{p+1}$ and so $\mathbb{U}_{p}^{-1} \mathbb{U}_{p+1}=U_{p+1}$ commutes with $R_{i_{p+1}}$; hence, $u_{p+1}$ commutes with $r_{i_{p+1}}$ and so $u_{p+1} \in\left\langle\operatorname{root}\left(r_{i_{p+1}}\right)\right\rangle$. Now $w^{\prime}$ can be obtained from $w$ by replacing the subword $t_{i_{p}}^{\varepsilon_{p}} u_{p+1} t_{i_{p+1}}^{\varepsilon_{p+1}}$ to $u_{p+1}$. But $\left(t_{i_{p}}^{\varepsilon_{p}} u_{p+1} t_{i_{p+1}}^{\varepsilon_{p+1}}\right)^{-1} u_{p+1} \in \mathcal{N}$ since $t_{i_{p+1}}$ commutes with $\operatorname{root}\left(r_{i_{p+1}}\right)$ modulo $\mathcal{N}$.

To see the first part of the claim, let us capitalize the $v_{i}$ 's:

$$
\begin{array}{ccc}
V_{1}=U_{1}, & & V_{p+1}=U_{p+3}, \\
\vdots & V_{p}=U_{p} U_{p+1} U_{p+2}, & \vdots \\
V_{p-1}=U_{p-1}, & & V_{l-1}=U_{l+1} .
\end{array}
$$


And let us compute the $\mathbb{V}_{i}=V_{1} V_{2} \ldots V_{i}$ 's:

$$
\begin{aligned}
& \mathbb{V}_{1}=\mathbb{U}_{1}, \\
& \mathbb{V}_{p+1}=\mathbb{U}_{p+3}, \\
& \mathbb{V}_{p}=\mathbb{U}_{p+2}, \\
& \mathbb{V}_{p-1}=\mathbb{U}_{p-1}, \\
& \mathbb{V}_{l-1}=\mathbb{U}_{l+1} .
\end{aligned}
$$

Finally, the identity among relations associated to $w^{\prime}$ is

$$
\begin{aligned}
\left(\mathbb{V}_{1} R_{i_{1}}^{\varepsilon_{1}} \mathbb{V}_{1}^{-1}=\mathbb{U}_{1} R_{i_{1}}^{\varepsilon_{1}} \mathbb{U}_{1}^{-1},\right. & \vdots \\
\mathbb{V}_{p-1} R_{i_{p-1}}^{\varepsilon_{p-1}} \mathbb{V}_{p-1}^{-1} & =\mathbb{U}_{p-1} R_{i_{p-1}}^{\varepsilon_{p-1}} \mathbb{U}_{p-1}^{-1}, \\
\mathbb{V}_{p} R_{i_{p+2}}^{\varepsilon_{p+2}} \mathbb{V}_{p}^{-1} & =\mathbb{U}_{p+2} R_{i_{p+2}}^{\varepsilon_{p+2}} \mathbb{U}_{p+2}^{-1}, \\
\mathbb{V}_{p+1} R_{i_{p+3}}^{\varepsilon_{p+3}} \mathbb{V}_{p+1}^{-1} & =\mathbb{U}_{p+3} R_{i_{p+3}+3}^{\varepsilon_{p+3}} \mathbb{U}_{p+3}^{-1}, \\
& \vdots \\
\mathbb{V}_{l-2} R_{i_{l}}^{\varepsilon_{l}} \mathbb{V}_{l-2}^{-1} & \left.=\mathbb{U}_{l} R_{i_{l}}^{\varepsilon_{l}} \mathbb{U}_{l}^{-1}\right),
\end{aligned}
$$

which coincides with the identity among relations obtained from (2) after applying the Peiffer transformation (4).

Case 3: Consider an insertion, and argue in a similar way as in Case 2.

This concludes the proof.

\section{A recursively presented orbit undecidable subgroup of $\operatorname{Aut}\left(F_{3}\right)$}

In [2], O. Bogopolski, A. Martino and E. Ventura studied the conjugacy problem for extensions of groups. In that context, the notion of orbit decidability is crucial and we recall it here.

Let $F$ be a group, and $A \leqslant \operatorname{Aut}(F)$. We say that $A$ is orbit decidable if and only if there exists an algorithm such that, given $u, v \in F$, decides whether $v$ is conjugate to $\alpha(u)$ for some $\alpha \in A$.

The main result in [2] states that, given a short exact sequence of groups

$$
1 \rightarrow F \rightarrow G \rightarrow P \rightarrow 1
$$

with some conditions on $F$ and $P$, the group $G$ has solvable conjugacy problem if and only if the action subgroup

$$
A_{G}=\left\{\gamma_{g}: F \rightarrow F, x \mapsto g^{-1} x g \mid g \in G\right\} \leqslant \operatorname{Aut}(F)
$$

is orbit decidable (see [2], Theorem 3.1, for details). 
In particular, this applies to the case where $F$ and $P$ are finitely generated free groups, giving a characterization of the solvability of the conjugacy problem within the family of [finitely generated free]-by-[finitely generated free] groups. This family of groups is interesting because C.F. Miller, back in the 1970s, already showed the existence of [finitely generated free]-by-[finitely generated free] groups with unsolvable conjugacy problem (see [13]). Via [2], Theorem 3.1, this can be restated by saying that $\operatorname{Aut}\left(F_{n}\right)$ contains finitely generated orbit undecidable subgroups (for some $n$ ).

Question 6 in the last section of [2] asks whether finitely presented subgroups $A \leqslant$ $\operatorname{Aut}\left(F_{n}\right)$ are orbit decidable or not. The answer is known to be positive in rank 2 (every finitely generated subgroup of $\operatorname{Aut}\left(F_{2}\right)$ is orbit decidable, see [2], Proposition 6.21), but open for bigger rank. The comment made in [2] after this question says that if $H$ is a finitely generated group with unsolvable word problem, then Mihailova's group $M(H)$ is isomorphic to an orbit undecidable subgroup of $\operatorname{Aut}\left(F_{3}\right)$. And, as mentioned in the introduction, this subgroup is then finitely generated, and recursively presented, but it cannot be finitely presented.

In the rest of the paper, we will recall how $M(H)$ can be embedded into $\operatorname{Aut}\left(F_{3}\right)$, in such a way that the image becomes an orbit undecidable subgroup of $\operatorname{Aut}\left(F_{3}\right)$. Then we will choose an appropriate $H$ and prove Theorem 4.2 by applying Theorem 1.1 to $A=M(H)$.

Of course, Theorem 4.2 does not answer the above mentioned Question 6, but shows its tightness in the sense that orbit undecidability is already showing up in the class of one-parametric recursively presented subgroups of $\operatorname{Aut}\left(F_{3}\right)$.

First, let $F_{3}=\langle q, a, b \mid\rangle$ be the free group on $\{q, a, b\}$, and let us embed $F_{2} \times F_{2}$ into $\operatorname{Aut}\left(F_{3}\right)$ in the following natural way. For every $u, v \in\langle a, b\rangle$, consider the automorphism

$$
u \theta_{v}: F_{3} \rightarrow F_{3}, \quad q \mapsto u q v, a \mapsto a, b \mapsto b .
$$

Clearly, $u_{1} \theta_{1} \cdot{ }_{u_{2}} \theta_{1}={ }_{u_{1}} u_{2} \theta_{1}$ and ${ }_{1} \theta_{v_{1}} \cdot{ }_{1} \theta_{v_{2}}={ }_{1} \theta_{v_{2} v_{1}}$, which means that $\left\{\theta_{u} \mid\right.$ $u \in\langle a, b\rangle\} \simeq F_{2}$ and $\left\{{ }_{1} \theta_{v} \mid v \in\langle a, b\rangle\right\} \simeq F_{2}^{\text {op }} \simeq F_{2}$. It is also clear that ${ }_{u} \theta_{1} \cdot{ }_{1} \theta_{v}=$ ${ }_{u} \theta_{v}={ }_{1} \theta_{v} \cdot{ }_{u} \theta_{1}$. So we have an embedding $\theta: F_{2} \times F_{2} \simeq F_{2}^{\mathrm{op}} \times F_{2}^{\mathrm{op}} \hookrightarrow \operatorname{Aut}\left(F_{3}\right)$ given by $(u, v) \mapsto u^{-1} \theta_{v}$, whose image is

$$
F_{2} \times F_{2} \simeq B=\left\langle a^{-1} \theta_{1}, b^{-1} \theta_{1},{ }_{1} \theta_{a},{ }_{1} \theta_{b}\right\rangle=\left\{u \theta_{v} \mid u, v \in\langle a, b\rangle\right\} \leqslant \operatorname{Aut}\left(F_{3}\right) .
$$

As shown in [2], Section 7.2, the element $q a q b q$ satisfies the technical condition required in [2], Proposition 7.3. Hence, we have

Lemma 4.1 (7.3 in [2]). For the above defined subgroup $B \leqslant \operatorname{Aut}\left(F_{3}\right)$ and for every subgroup $A \leqslant B$, undecidability of the membership problem for $A$ in $B$ implies orbit undecidability for $A$ in $\operatorname{Aut}\left(F_{3}\right)$.

We are ready to deduce the main result of this section. 
Theorem 4.2. There exists a finitely generated (and not finitely presented) orbit undecidable subgroup $A \leqslant \operatorname{Aut}\left(F_{3}\right)$ admitting a one-parametric recursive presentation as in Theorem 1.1.

Proof. In [7], D. J. Collins and C. F. Miler III proved that there exists a finite, concise and Peiffer aspherical presentation $\left\langle x_{1}, \ldots, x_{n} \mid R_{1}, \ldots, R_{m}\right\rangle$ of a group $H$ with unsolvable word problem. The corresponding Mihailova's group $M(H)$ is a subgroup of $F_{n} \times F_{n}$ and the membership problem for $M(H)$ in $F_{n} \times F_{n}$ is unsolvable.

Now, denoting $A=M(H)$ and using a finite index embedding of $F_{n} \times F_{n}$ in $B \cong F_{2} \times F_{2}$, we have that $A \leqslant B$ and the membership problem for $A$ in $B$ is unsolvable. By Lemma 4.1, $A$ is an orbit undecidable subgroup of $\operatorname{Aut}\left(F_{3}\right)$.

Moreover, as it was discussed in the introduction, $A$ is finitely generated, and is not finitely presented. But Theorem 1.1 provides an explicit one-parametric recursive presentation for $A$. This concludes the proof.

We end by reproducing [2], Question 6, again:

Question 4.3. Does there exist a finitely presented orbit undecidable subgroup of $\operatorname{Aut}\left(F_{n}\right)$ for $n \geqslant 3$ ?

Acknowledgements. The first named author thanks the MPIM at Bonn for its support and excellent working conditions during the fall 2008, while this research was finished. The second author gratefully acknowledges partial support from the MEC (Spain) and the EFRD (EC) through project number MTM-2008-01550.

\section{References}

[1] G. Baumslag and J. E. Roseblade, Subgroups of direct products of free groups. J. London Math. Soc. (2) 30 (1984), 44-52. Zbl 0559.20018 MR 760871

[2] O. Bogopolski, A. Martino, and E. Ventura, Orbit decidability and the conjugacy problem for some extensions of groups. Trans. Amer. Math. Soc. 362 (2010), 2003-2036. Zbl 05692501 MR 2574885

[3] W. W. Boone, The word problem. Ann. of Math. (2) 70 (1959), 207-265. Zbl 0102.00902 MR 0179237

[4] V. V. Borisov, Simple examples of groups with unsolvable word problem. Mat. Zametki 6 (1969), 521-532; English transl. Math. Notes 6 (1969), 768-775. Zbl 0215.10803 MR 0260851

[5] M. R. Bridson and D. T. Wise, $\mathcal{V} \mathscr{H}$ complexes, towers and subgroups of $F \times F$. Math. Proc. Cambridge Philos. Soc. 126 (1999), 481-497. Zbl 0942.20009 MR 1684244

[6] I. M. Chiswell, D. J. Collins, and J. Huebschmann, Aspherical group presentations. Math. Z. 178 (1981), 1-36. Zbl 0443.20030 MR 627092 
[7] D. J. Collins and C. F. Miller, III, The word problem in groups of cohomological dimension 2. In Groups St. Andrews 1997 in Bath, I, London Math. Soc. Lecture Note Ser. 260, Cambridge University Press, Cambridge 1999, 211-218. Zbl 1001.20030 MR 1676618

[8] R. Grigorchuk, Solved and unsolved problems around one group. In Infinite groups: geometric, combinatorial and dynamical aspects, Progr. Math. 248, Birkhäuser, Basel 2005, 117-218. Zbl 1165.20021 MR 2195454

[9] F. J. Grunewald, On some groups which cannot be finitely presented. J. London Math. Soc. (2) 17 (1978), 427-436. Zbl 0385.20020 MR 500627

[10] F. Grunewald and V. Platonov, Rigidity results for groups with radical cohomology of finite groups and arithmeticity problems. Duke Math. J. 100 (1999), 321-358. Zbl 1007.11029 MR 1722957

[11] R. C. Lyndon and P. E. Schupp, Combinatorial group theory. Ergeb. Math. Grenzgeb. 89, Springer-Verlag, Berlin 1977. Zbl 0368.20023 MR 0577064

[12] K. A. Mihaullova, The occurrence problem for direct products of groups. Dokl. Akad. Nauk SSSR 119 (1958), 1103-1105 (in Russian). MR 0100018

[13] C. F. Miller III, On group-theoretic decision problems and their classification. Ann. of Math. Stud. 68, Princeton University Press, Princeton 1971. Zbl 0277.20054 MR 0310044

[14] P. S. Novikov, On the algorithmic insolvability of the word problem in group theory. Trudy Mat. Inst. Steklov. 44 1955, 1-143; English transl. in Amer. Math. Soc. Transl. (2) 9, Amer. Math. Soc., Providence, R.I., 1958, 1-122. Zbl 0093.01304 MR 0075197

[15] H. Short, Finitely presented subgroups of a product of two free groups. Q. J. Math. 52 (2001), 127-131. Zbl 0984.20021 MR 1820907

Received October 15, 2008; revised August 10, 2009

O. Bogopolski, Mathematisches Institut der Heinrich-Heine-Universität Düsseldorf, Universitätsstr. 1, 40225 Düsseldorf, Germany, and Institute of Mathematics of Siberian Branch of Russian Academy of Sciences, Koptjuga 4, Novosibirsk 630090, Russia E-mail: Oleg_Bogopolski@yahoo.com

E. Ventura, Department of Applied Mathematics III, Escola Politècnica Superior d'Enginyeria de Manresa, Universitat Politècnica de Catalunya, Av. Bases de Manresa 61-73, 08242 Manresa, Barcelona, Catalonia, Spain

E-mail: enric.ventura@upc.edu 